\title{
Error resilient non-asymmetric Slepian-Wolf Coding
}

\author{
C. Herzet, V. Toto-Zarasoa, A. Roumy \\ INRIA-Rennes, Campus de Beaulieu, 35042 Rennes Cedex, France \\ Email: cherzet@irisa.fr
}

\begin{abstract}
We consider non-asymmetric distributed source coding (DSC) that achieves any point in the Slepian-Wolf (SW) region. We study the error propagation phenomena and propose a decoding algorithm which limits this phenomena. For the case of turbo-codes, design rules are derived in order for the decoder to recover the sources.
\end{abstract}

Distributed source coding (DSC) refers to the problem of compressing two or more correlated sources, where the encoding is performed separately but the decoding jointly. This setup is usually referred to as Slepian-Wolf coding (SW), since in [10], Slepian and Wolf derived the theoretical limits of the lossless coding of two correlated memoryless sources. These theoretical limits are given in terms of a set of achievable compression rates, also called the $S W$ region. For two discrete sources, [10] shows the surprising result that the sum compression-rate of the two sources experiences no loss when the encoders cannot communicate. This property is very valuable in the context of sensor networks, where communication between the sensors can be saved. Moreover, in this context it is desirable to design a SW codec, that can adapt the compression rates of the sources to the channel between the sensors and the fusion center. Therefore, we consider in this paper SW codes that can achieve any point of the SW region.

Moreover, Wyner showed in [14] the optimality of binary linear codes to achieve the corner points of the SW region for two correlated binary sources, in a scheme called the syndrome approach. Due to its optimality, we also consider this approach here. First practical syndrome solutions appeared in [4] for systematic convolutional codes and in [3] for LDPC codes. We rather consider in this paper the syndrom code proposed in [7] since it uses any convolutional (not only systematic) code or any turbo code and since it can also adapt to any correlation between the sources.

In order to achieve any point of the SW region, [9], [2], [12] propose a two step scheme, where first the difference between the sources is estimated and then each source is recovered. If the difference contains errors, this may yield error propagation. In this paper, we analyze this error propagation phenomena and propose solutions in order to avoid it.

\footnotetext{
${ }^{1}$ This research was partially supported by the French National Research Agency (ANR) under the Essor project and by the French European Commission in the framework of the FP7 Network of Excellence in Wireless COMmunications NEWCOM++ (contract n.216715).
}

\section{DSC: THEORETICAL RESULTS AND OPTIMAL CODES.}

\section{A. Information theoretical results}

In SW coding, two discrete memoryless sources $X$ and $Y$ are separately encoded but jointly decoded (see Figure 1-left). The set of achievable compression rates $\left(R_{X}, R_{Y}\right)$, also called the $S W$ region [10], is an unbounded polygon with two corner points (see Figure 1-right).

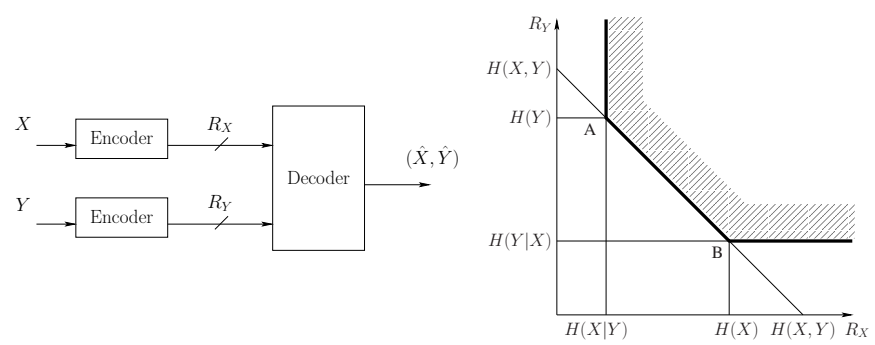

Fig. 1. Distributed source coding of statistically dependent i.i.d. discrete random sequences $X$ and $Y$. Set-up (left); achievable rate region (right).

At these corner points, one source (say $Y$ for point A) is compressed at its entropy rate $H(Y)$, whereas $X$ is compressed at the conditional entropy $H(X \mid Y)$. The sources $X$ and $Y$ play different roles, and therefore the scheme operating at a corner point of the SW region is usually referred to as asymmetric $S W$ coding. If both sources are compressed in order to reach any point of the segment between $A$ and $B$ in Figure 1, then the scheme is called non asymmetric. In this paper, we consider rate adaptive non asymmetric SW codes in the sense that the same code can achieve any rate on the dominant face of the SW region (segment between A and B).

\section{B. Practical syndrome-based SW coding}

In [14], Wyner showed that binary linear codes can achieve optimality for the problem of asymmetric SW coding. Recently, a number of practical approaches based on linear codes have therefore been proposed. In [4], [13], the authors considered the asymmetric SW problem based on systematic convolutional codes and systematic turbo codes whereas LDPC codes were used in [3]. In the non-asymmetric setup, an approach based on the partition of a code is presented in [5], [11], whereas the original code is used in [2], [12]. In this paper, we consider the non-asymmetric technique in [2] based on the SW scheme proposed in [7].

In order to present the scheme considered in this paper, we first review some definitions and properties on binary linear codes. A binary $(n, k)$ code $\mathcal{C}$ is defined by an $(n-k) \times n$ 
parity-check matrix $\mathbf{H}$, and contains all the $n$-length vectors ${ }^{1}$ x s. t. $\mathbf{H x}=\mathbf{0}$ :

$$
\mathcal{C}=\{\mathbf{x}: \mathbf{H x}=\mathbf{0}\} .
$$

The code partitions the space containing $2^{n}$ sequences into $2^{n-k}$ cosets of $2^{k}$ words with maximum Hamming distance. Each coset is indexed by an $(n-k)$-length syndrome, defined as $\mathbf{s}=\mathbf{H x}$. In other words, all sequences in a coset share the same syndrome: $\mathcal{C}_{\mathbf{s}}=\{\mathbf{x}: \mathbf{H x}=\mathbf{s}\}$.

Let $\mathbf{x}$ and $\mathbf{y}$ be two correlated binary sequences of length $n$. These sequences are the realizations of the sources $X$ and $Y$. The scheme considered in [2] uses the $(n-k) \times n$ paritycheck matrix $\mathbf{H}$ defined above and a design parameter $k^{\prime} \in$ $[0, k]$, that allows to vary the compression rates $\left(R_{X}, R_{Y}\right)$. The two correlated binary sequences of length $n, \mathbf{x}$ and $\mathbf{y}$, are split into three parts. More precisely, $\mathbf{x}=\left(\mathbf{x}_{1}^{k^{\prime}}, \mathbf{x}_{k^{\prime}+1}^{k}, \mathbf{x}_{k+1}^{n}\right)$ and $\mathbf{y}=\left(\mathbf{x}_{1}^{k^{\prime}}, \mathbf{y}_{k^{\prime}+1}^{k}, \mathbf{y}_{k+1}^{n}\right)$. The syndromes $\mathbf{s}_{x}=\mathbf{H x}$ and $\mathbf{s}_{y}=\mathbf{H y}$, of length $(n-k)$, are computed for both sequences and transmitted to the decoder. In addition, the $k^{\prime}$ first bits of $\mathbf{x}, \mathbf{x}_{1}^{k^{\prime}}$, and the $k-k^{\prime}$ next bits for the source $\mathbf{y}, \mathbf{y}_{k^{\prime}+1}^{k}$, are transmitted as systematic bits. The total rate for the sequences $\mathbf{x}$ and $\mathbf{y}$ of length $n$ is respectively $n-k+k^{\prime}$ and $n-k^{\prime}$ bits. The structure of the coders is depicted in Figure 2.

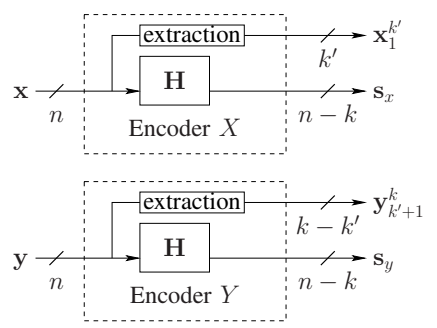

Fig. 2. The non asymmetric coders in the syndrome approach [2].

At the receiver side, the decoder performs a suboptimal decoding as follows. It first estimates the error pattern $\mathbf{z}=\mathbf{x} \oplus \mathbf{y}$ as the minimum weight vector with syndrome $\mathbf{s}_{z}=\mathbf{s}_{x} \oplus \mathbf{s}_{y}$. Once the error pattern is found, the subsequences of informa-

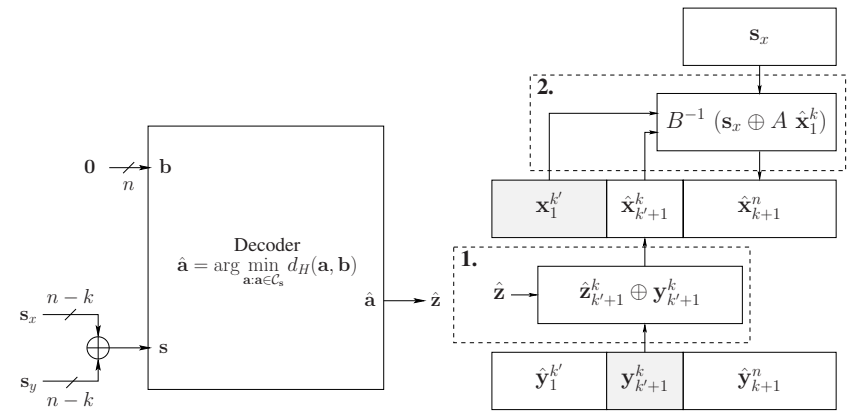

Fig. 3. Non-asymmetric decoder: estimation of the error pattern $\mathbf{z}=\mathbf{x} \oplus \mathbf{y}$ (left); reconstruction of the source sequence $\mathbf{x}$ (right).

tion bits $\mathbf{x}_{k^{\prime}+1}^{k}$ and $\mathbf{y}_{1}^{k^{\prime}}$ are retrieved from the error pattern by

\footnotetext{
${ }^{1}$ All vectors are column vectors. Moreover, $\oplus$ denotes the addition over the finite field of order 2 and + the addition over the real field.
}

taking into account that $\mathbf{z}=\mathbf{x} \oplus \mathbf{y}$ i.e., (see Figure 3-right):

$$
\hat{\mathbf{x}}_{k^{\prime}+1}^{k}=\mathbf{y}_{k^{\prime}+1}^{k} \oplus \hat{\mathbf{z}}_{k^{\prime}+1}^{k} \text { and } \hat{\mathbf{y}}_{1}^{k^{\prime}}=\mathbf{x}_{1}^{k^{\prime}} \oplus \hat{\mathbf{z}}_{1}^{k^{\prime}} .
$$

Subsequences of $n-k$ bits, i.e. $\mathbf{x}_{k+1}^{n}$ and $\mathbf{y}_{k+1}^{n}$, remain to be computed for both sequences. Let us assume that $\mathbf{H}=$ $(\mathbf{A} \mathbf{B})$, where $\mathbf{B}$ is an invertible square matrix of dimension $(n-k) \times(n-k)$. Such a decomposition (up to a permutation on the columns) exists since $\mathbf{H}$ has rank $n-k$. Thus, $\mathbf{s}_{x}=\mathbf{H x}=$ (A B ) $\mathbf{x}=A \mathbf{x}_{1}^{k} \oplus \mathbf{B} \mathbf{x}_{k+1}^{n}$, and the remaining $n-k$ unknown bits of the sequence $\mathbf{x}$ (and similarly for the sequence $\mathbf{y}$ ) can be computed as

$$
\hat{\mathbf{x}}_{k+1}^{n}=\mathbf{B}^{-1}\left(\mathbf{s}_{x} \oplus \mathbf{A} \hat{\mathbf{x}}_{1}^{k}\right),
$$

where $\mathbf{B}^{-1}$ denotes the inverse of the matrix $\mathbf{B}$ [2]. The authors in [2] have used LDPC codes.

\section{Robust SW CODE: The CONVOLUtional CASE}

If the error pattern is not perfectly estimated, the estimation of the remaining symbols $\mathbf{x}_{k+1}^{n}$ in equation (2) may yield error propagation. In the rest of this paper, we analyze the error propagation phenomena and propose solutions to limit it.

A first solution to limit error propagation is based on the fact that the unknown positions in the vector $\mathrm{x}$ are design parameters. Therefore, these positions (or equivalently the columns of the matrix $\mathbf{H}$ to be extracted in order to build the matrix $\mathbf{B}$ ) can be chosen such that the inverse $\mathbf{B}^{-1}$ is as sparse as possible. Figure 4 shows the BER of the error pattern $\mathbf{z}$ (continuous line) and its effect on the estimation of the source sequence $\mathrm{x}$. Performance is shown for a convolutional code. The dotted curve represents the BER, when equation (2) is performed with an arbitrary invertible matrix $\mathbf{B}$. This BER can be lowered to the dashed curve, if $\mathbf{B}$ is chosen with an inverse as sparse as possible.

In order to further limit the error propagation phenomena, we consider a "soft" decoding procedure to estimate the $n-k$ unknown bits of $\mathbf{x}$ and $\mathbf{y}$. To be more precise, consider the extended alphabet $\{0,1, \epsilon\}$, where $\epsilon$ denotes the event "erasure" and let $p=\operatorname{BER}(\mathbf{z})$. Let us then define an observation vector $\mathbf{w}=\left(w_{1}, \ldots, w_{n}\right)^{T}$ as follows:

$$
w_{i}= \begin{cases}x_{i} & \text { if } i=1, \ldots, k^{\prime}, \\ y_{i} \oplus \hat{z}_{i} & \text { if } i=k^{\prime}+1, \ldots, k, \\ \epsilon & \text { if } i=k+1, \ldots, n,\end{cases}
$$

where $\hat{z}_{i}$ is assumed to be Bernoulli distributed with parameter $p$. We are then looking for the solution of the following optimization problem

$$
\hat{\mathbf{x}}=\arg \max _{\tilde{\mathbf{x}}} p(\tilde{\mathbf{x}} \mid \mathbf{w})=\arg \max _{\tilde{\mathbf{x}}} p(\mathbf{w} \mid \tilde{\mathbf{x}}) p(\tilde{\mathbf{x}}),
$$

where $\tilde{\mathbf{x}}$ is a trial vector and

$$
p(\tilde{\mathbf{x}})= \begin{cases}2^{-k} & \forall \tilde{\mathbf{x}} \in \mathcal{C}_{\mathbf{s}_{x}} \\ 0 & \text { otherwise. }\end{cases}
$$

In the case of convolutional codes, this optimization can be efficiently implemented by a Viterbi decoder matched to a channel combining a perfect channel (for $\mathbf{x}_{1}^{k^{\prime}}$ ), a BSC (for $\hat{\mathbf{x}}_{k^{\prime}+1}^{k}$ with cross over probability $\left.p=\operatorname{BER}(\mathbf{z})\right)$ and a binary 


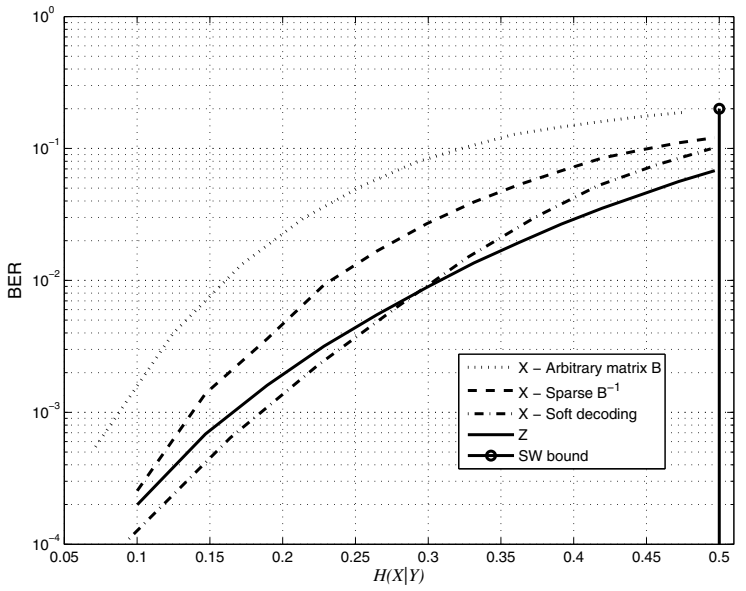

Fig. 4. Error propagation in the estimation of the source $X$. The convolutional code is defined by the following parity-check polynomials $\left(\begin{array}{lll}11 & 15 & 06 \\ 15 & 12 & 17\end{array}\right)$ and is punctured over a period of four trellis sections in order to get a $2: 1$ compression rate for the source.

erasure channel (BEC) with erasure probability $p_{\epsilon}=1$ (for $\mathbf{x}_{k+1}^{n}$ ). The advantage of this approach is that, rather than estimating $\mathbf{x}_{k+1}^{n}$ from "hard" decisions on $\mathbf{x}_{k^{\prime}+1}^{k}$, it performs a "soft" decoding taking into account the non-perfect nature of $\hat{\mathbf{x}}_{k^{\prime}+1}^{k}$. The performance achieved by this approach are represented by the dashed-dotted curve in Fig. 4. Interestingly, we see that it allows a significant improvement over the classical "hard-decision" approach. In particular, the BER of $\mathbf{x}$ remains almost the same as that of $\mathbf{z}$.

\section{ROBUST SW CODE: THE TURBO-CODE CASE}

Motivated by the good performance of the "soft" decoding (4) for convolutional codes, we turn ourselves to the more involved case of parallel-concatenated codes, like for example turbo codes. Although these codes exhibit very good distance properties, their structure does unfortunately not allow for tractable exact resolution of (4). Therefore, suboptimal iterative (turbo) decoding has to be considered. In this section, we will emphasize that this suboptimal decoding procedure may lead to significant degradations if the constituent codes are not designed carefully.

This section is organized as follows. In section III-A, we study under which conditions a maximum a posteriori (MAP) bit decoder can recover an erased bit. Then, in section III-B we derive conditions such that a suboptimal MAP bit decoder can recover $n-k$ erased bits. Finally, in section III-C, we propose a SW coding procedure based on turbo codes which is suited to the soft decoding of the last $n-k$ bits of $\mathbf{x}$ and y.

\section{A. Conditions on the parity-check matrix for erasure recovery under MAP decoding}

In this section, we give necessary and sufficient conditions on the parity-check matrix $\mathbf{H}$ such that a set of erased bits can be recovered under MAP decoding.

In the sequel, we will use the following notations: $w$ is the observation of $\mathrm{x}$ through a BEC; $\mathcal{E}$ is the set of indices of erasures in w, i.e., $\mathcal{E} \triangleq\left\{i \mid w_{i}=\epsilon\right\}$ and $\overline{\mathcal{E}} \triangleq\{1, \ldots, n\} \backslash \mathcal{E}$; $|\mathcal{E}|$ denotes the cardinality of $\mathcal{E} ; \mathbf{x}_{\mathcal{E}}$ (resp. $\mathbf{x}_{\overline{\mathcal{E}}}$ ) is the vector formed by the concatenation of the $x_{i}$ 's such that $i \in \mathcal{E}$ (resp. $i \in \overline{\mathcal{E}}) ; \mathbf{h}_{i}$ denotes the $i$ th column of $\mathbf{H} ; \mathbf{H}_{\mathcal{E}}\left(\right.$ resp. $\left.\mathbf{H}_{\overline{\mathcal{E}}}\right)$ is the matrix made up of the columns of $\mathbf{H}$ indexed by $\mathcal{E}$ (resp. by $\overline{\mathcal{E}}) ; \operatorname{col}\left(\mathbf{H}_{\mathcal{E}}\right)=\left\{\mathbf{h}_{i}\right\}_{i \in \mathcal{E}}$ is the set of columns of $\mathbf{H}_{\mathcal{E}}$; $\operatorname{span}\left(\operatorname{col}\left(\mathbf{H}_{\mathcal{E}}\right)\right)$ is the subspace generated by the columns of $\mathbf{H}_{\mathcal{E}}$ and $\operatorname{rank}\left(\mathbf{H}_{\mathcal{E}}\right)$ is its dimension; $\delta(x)$ is the Kronecker delta which is equal to one if $x=0$ and zero otherwise.

The main result of this section is stated in proposition 3.3. Our derivations are based on standard coding theory results that we reformulate here for the sake of completeness:

Proposition 3.1 (Sequence a posteriori probability): Let

$$
\mathcal{X}_{\mathcal{E}} \triangleq\left\{\tilde{\mathbf{x}} \in \mathcal{C}: \mathbf{H}_{\mathcal{E}} \tilde{\mathbf{x}}_{\mathcal{E}}=\mathbf{H}_{\overline{\mathcal{E}}} \mathbf{w}_{\overline{\mathcal{E}}}, \tilde{\mathbf{x}}_{\overline{\mathcal{E}}}=\mathbf{w}_{\overline{\mathcal{E}}}\right\}
$$

be the set of codewords $\tilde{\mathbf{x}}$ compatible with the received observations $\mathbf{w}$. Then, the codeword posterior probability can be expressed as

$$
p(\tilde{\mathbf{x}} \mid \mathbf{w})= \begin{cases}\frac{1}{\mid \mathcal{X}} \mid & \text { if } \tilde{\mathbf{x}} \in \mathcal{X}_{\mathcal{E}} \\ 0, & \text { otherwise }\end{cases}
$$

Moreover, the MAP block decoding recovers the codeword uniquely (i.e. $\left|\mathcal{X}_{\mathcal{E}}\right|=1$ ) if and only if $\operatorname{rank}\left(\mathbf{H}_{\mathcal{E}}\right)=|\mathcal{E}|$.

Proposition 3.2 (Bit a posteriori probability): $\forall i \in \mathcal{E}$, we have

$$
p\left(\tilde{x}_{i} \mid \mathbf{w}\right)= \begin{cases}\frac{1}{2} & \text { if } \mathbf{h}_{i} \in \operatorname{span}\left(\operatorname{col}\left(\mathbf{H}_{\mathcal{E}}\right) \backslash\left\{\mathbf{h}_{i}\right\}\right), \\ \delta\left(\tilde{x}_{i}-x_{i}\right) & \text { otherwise, }\end{cases}
$$

i.e., MAP bit decoding can recover an erased bit at position $i$ if and only if all the codewords $\tilde{\mathbf{x}} \in \mathcal{X}_{\mathcal{E}}$ are such that $\tilde{x}_{i}=x_{i}$.

Proof: We refer the reader to [6] for a proof of propositions 3.1 and 3.2.

Proposition 3.2 basically states that MAP bit decoding in a BEC leads to two possibilities: either we recover perfectly the erased bit (i.e., $p\left(\tilde{x}_{i} \mid \mathbf{w}\right)=\delta\left(\tilde{x}_{i}-x_{i}\right)$ ) or we do not increase at all the amount of information we have about it (i.e., $\left.p\left(\tilde{x}_{i} \mid \mathbf{y}\right)=1 / 2\right)$. Hence, for a given $\mathbf{H}_{\mathcal{E}}$, we can wonder how many bits we can recover by MAP bit decoding. The next proposition answers this question.

Proposition 3.3: Let $\mathcal{R} \subseteq \mathcal{E}$ be the set of indices of the erased bits that can be recovered by MAP bit decoding, i.e.,

$$
\mathcal{R}=\left\{i \in \mathcal{E}: p\left(\tilde{x}_{i} \mid \mathbf{w}\right)=\delta\left(\tilde{x}_{i}-x_{i}\right)\right\} .
$$

Let moreover $\mathcal{B}$ be such that $\left\{\mathbf{h}_{i}\right\}_{i \in \mathcal{B}}$ is a basis of $\operatorname{span}\left(\operatorname{col}\left(\mathbf{H}_{\mathcal{E}}\right)\right)$ and let $\mathrm{nz}\left(\mathbf{H}_{\mathcal{E}}\right) \triangleq\left\{i \in \mathcal{E} \backslash \mathcal{B} \mid \mathbf{h}_{i} \neq \mathbf{0}\right\}$. We have

$$
|\mathcal{R}| \leq \operatorname{rank}\left(\mathbf{H}_{\mathcal{E}}\right)
$$

with equality if and only if $n z\left(\mathbf{H}_{\mathcal{E}}\right)=\emptyset$. In other words, equality holds in (9) if and only if all the columns of $\mathbf{H}_{\mathcal{E}}$ which do not belong to the basis $\left\{\mathbf{h}_{i}\right\}_{i \in \mathcal{B}}$ are equal to zero.

Proof: Since $\left\{\mathbf{h}_{j}\right\}_{j \in \mathcal{B}}$ is a basis, $\mathbf{h}_{i} \in \operatorname{span}\left(\left\{\mathbf{h}_{j}\right\}_{j \in \mathcal{B}}\right)=$ $\operatorname{span}\left(\operatorname{col}\left(\mathbf{H}_{\mathcal{E}}\right) \backslash\left\{\mathbf{h}_{i}\right\}\right) \forall i \in \mathcal{E} \backslash \mathcal{B}$. Therefore, from proposition 
3.2 we cannot recover $x_{i}$ by MAP bit decoding $\forall i \in \mathcal{E} \backslash \mathcal{B}$. This implies that $|\mathcal{R}| \leq|\mathcal{B}|=\operatorname{rank}\left(\mathbf{H}_{\mathcal{E}}\right)$.

We now prove that $\mathrm{nz}\left(\mathbf{H}_{\mathcal{E}}\right)=\emptyset$ is a necessary and sufficient condition for the equality to hold in (9). On the one hand, if $\mathrm{nz}\left(\mathbf{H}_{\mathcal{E}}\right)=\emptyset$, we have $\forall i \in \mathcal{B}$ :

$$
\operatorname{span}\left(\operatorname{col}\left(\mathbf{H}_{\mathcal{E}}\right) \backslash\left\{\mathbf{h}_{i}\right\}\right)=\operatorname{span}\left(\left\{\mathbf{h}_{j}\right\}_{j \in \mathcal{B}} \backslash\left\{\mathbf{h}_{i}\right\}\right) .
$$

Now, since $\left\{\mathbf{h}_{j}\right\}_{j \in \mathcal{B}}$ is a basis, $\mathbf{h}_{i} \notin \operatorname{span}\left(\left\{\mathbf{h}_{j}\right\}_{j \in \mathcal{B}} \backslash\left\{\mathbf{h}_{i}\right\}\right)$ and, from proposition 3.2, $x_{i}$ can be recovered $\forall i \in \mathcal{B}$. Therefore, $|\mathcal{R}|=|\mathcal{B}|=\operatorname{rank}\left(\mathbf{H}_{\mathcal{E}}\right)$.

On the other hand, if $\operatorname{nz}\left(\mathbf{H}_{\mathcal{E}}\right) \neq \emptyset$, we have $|\mathcal{R}|<$ $\operatorname{rank}\left(\mathbf{H}_{\mathcal{E}}\right)$. If $i \in \mathrm{nz}\left(\mathbf{H}_{\mathcal{E}}\right), \mathbf{h}_{i} \neq \mathbf{0}$ and $\exists \mathcal{S}_{i} \subseteq \mathcal{B}$ such that $\mathbf{h}_{i}=\sum_{j \in \mathcal{S}_{i}} \mathbf{h}_{j}$. Hence, we have $\forall j \in \mathcal{S}_{i}$ that

$$
\mathbf{h}_{j} \in \operatorname{span}\left(\left\{\mathbf{h}_{l}\right\}_{l \in \mathcal{S}_{i} \backslash\{j\}} \cup\left\{\mathbf{h}_{i}\right\}\right) .
$$

Now,

$$
\operatorname{span}\left(\left\{\mathbf{h}_{l}\right\}_{l \in \mathcal{S}_{i} \backslash\{j\}} \cup\left\{\mathbf{h}_{i}\right\}\right) \subseteq \operatorname{span}\left(\operatorname{col}\left(\mathbf{H}_{\mathcal{E}}\right) \backslash\left\{\mathbf{h}_{j}\right\}\right) .
$$

From proposition 3.2, this implies that $p\left(x_{i} \mid \mathbf{w}\right)=1 / 2 \forall i \in$ $\mathcal{S}_{i}$. Hence,

$$
|\mathcal{R}|=\operatorname{rank}\left(\mathbf{H}_{\mathcal{E}}\right)-\left|\cup_{i \in \mathrm{nz}\left(\mathbf{H}_{\mathcal{E}}\right)} \mathcal{S}_{i}\right| .
$$

Since $\left|\mathcal{S}_{i}\right| \geq 1$, we obtain $|\mathcal{R}|<\operatorname{rank}\left(\mathbf{H}_{\mathcal{E}}\right)$.

Proposition 3.3 gives necessary and sufficient conditions for recovering $\operatorname{rank}\left(\mathbf{H}_{\mathcal{E}}\right)$ erased bits when MAP bit decoding is performed. In the next section, we will see that this condition turns out to be key in the design of "good" codes for distributed source coding when suboptimal decoding is considered.

\section{B. Design of codes suited to "2-step" decoding}

In the previous section, we saw that all the erased bits can be recovered by a MAP bit decoding if and only if $\operatorname{rank}\left(\mathbf{H}_{\mathcal{E}}\right)=$ $n-k$. In this section, we investigate under which conditions, the $n-k$ erased bits can still be recovered under a particular suboptimal decoding procedure.

We consider an overall code $\mathcal{C}$ which is made up by the parallel concatenation of two codes $\mathcal{C}^{(1)}$ and $\mathcal{C}^{(2)}$. We denote $\mathbf{H}^{(1)}\left(\right.$ resp. $\left.\mathbf{H}^{(2)}\right)$ the $\frac{n-k}{2} \times n$ parity-check matrix defining $\mathcal{C}^{(1)}$ (resp. $\mathcal{C}^{(2)}$ ). We assume that $\mathbf{x} \in \mathcal{C}$ is transmitted through a BEC and $w$ is the observation vector. Let us define

$$
p^{(l)}\left(\tilde{x}_{i}=b \mid \mathbf{w}\right) \triangleq \frac{\sum_{\tilde{\mathbf{x}} \in \mathcal{X}_{i}^{(l)}(b)} p(\mathbf{w} \mid \tilde{\mathbf{x}}) p^{(l)}(\tilde{\mathbf{x}})}{\sum_{\tilde{\mathbf{x}} \in \mathcal{C}^{(l)}} p(\mathbf{w} \mid \tilde{\mathbf{x}}) p^{(l)}(\tilde{\mathbf{x}})},
$$

where

$$
\begin{aligned}
& p^{(l)}(\tilde{\mathbf{x}}) \triangleq \begin{cases}2^{-\frac{n+k}{2}} & \text { if } \tilde{\mathbf{x}} \in \mathcal{C}^{(l)}, \\
0 & \text { otherwise, }\end{cases} \\
& \mathcal{X}_{i}^{(l)}(b) \triangleq\left\{\tilde{\mathbf{x}} \in \mathcal{C}^{(l)} \mid \tilde{x}_{i}=b\right\}, \quad b \in\{0,1\} .
\end{aligned}
$$

Based on this definition, we define a "2-step" decoding procedure as follows:

- Step 1: Compute $p^{(1)}\left(\tilde{x}_{i} \mid \mathbf{w}\right) \forall i$ and define

$$
w_{i}^{\prime} \triangleq \begin{cases}x_{i} & \text { if } p^{(1)}\left(\tilde{x}_{i} \mid \mathbf{w}\right)=\delta\left(\tilde{x}_{i}-x_{i}\right), \\ w_{i} & \text { otherwise. }\end{cases}
$$

- Step 2: Compute $\hat{x}_{i}$ as follows

$$
\hat{x}_{i}=\arg \max _{\tilde{x}_{i}} p^{(2)}\left(\tilde{x}_{i} \mid \mathbf{w}^{\prime}\right) .
$$

In words, the procedure described above is as follows: we first perform a MAP bit decoding by only taking $\mathcal{C}^{(1)}$ into account. If $\mathcal{C}^{(1)}$ is such that some erased bits can be recovered, the observation vector is modified by replacing the erased bits by their actual value. This leads to $\mathbf{w}^{\prime}$. We then perform a second MAP bit decoding by only taking $\mathcal{C}^{(2)}$ and the new observation vector $\mathbf{w}^{\prime}$ into account.

It is interesting to notice that the "2-step" procedure described above is in fact a particular case of turbo decoding when the channel is a BEC. Indeed, in this case the loglikelihood ratios (LLRs) exchanged by the constituent decoders of a turbo decoder are either equal to 0 or $\pm \infty$. The modification of the observation vector $\mathbf{w}$ into $\mathrm{w}^{\prime}$ in the " 2 step" procedure is therefore strictly equivalent to feeding the LLRs computed by the first constituent decoder to the second one.

We now investigate under which conditions the "2-step" decoding procedure can recover $n-k$ erased bits. In the sequel, we will assume without loss of generality that the erased bits correspond to the last $n-k$ bits of $\mathrm{x}$ (this can always be achieved by a permutation of the elements of $\mathbf{x}$ ). Therefore,

$$
\mathbf{H}=\left[\mathbf{H}_{\overline{\mathcal{E}}} \mathbf{H}_{\mathcal{E}}\right]=\left[\begin{array}{cc}
\mathbf{H}_{\overline{\mathcal{E}}}^{(1)} & \mathbf{H}_{\mathcal{E}}^{(1)} \\
\mathbf{H}_{\overline{\mathcal{E}}}^{(2)} & \mathbf{H}_{\mathcal{E}}^{(2)}
\end{array}\right] .
$$

In the following proposition, we give necessary and sufficient conditions such that the "2-step" procedure can recover all the $n-k$ erased bits:

Proposition 3.4: Assume $\operatorname{rank}\left(\mathbf{H}_{\mathcal{E}}\right)=n-k$. Let $\mathbf{H}_{\pi(\mathcal{E})}$ be a matrix obtained by a permutation $\pi$ of the columns of $\mathbf{H}_{\mathcal{E}}$. Then, the "2-step" decoding procedure can recover $n-k$ erased bits if and only if there exists a permutation $\pi$ such that $\mathbf{H}_{\pi(\mathcal{E})}$ has the following structure:

$$
\mathbf{H}_{\pi(\mathcal{E})}=\left[\begin{array}{cc}
\mathbf{A} & \mathbf{0}_{\frac{n-k}{2}} \\
\mathbf{B} & \mathbf{C}
\end{array}\right],
$$

where $\mathbf{0}_{\frac{n-k}{2}}$ denotes a $\left(\frac{n-k}{2}, \frac{n-k}{2}\right)$ zero matrix. In other words, $\frac{n-k}{2}$ columns of $\mathbf{H}_{\mathcal{E}}^{(1)}$ must be equal to zero.

Proof: Let $\mathcal{R}^{(1)}$ (resp. $\mathcal{R}^{(2)}$ ) be the set of indices of the bits recovered by the first (resp. second) decoder. Note that we have $\mathcal{R}^{(1)} \cap \mathcal{R}^{(2)}=\emptyset$ since the bits recovered by the first decoder need no longer to be recovered by the second. Then, the total number of erased bits recovered by the " 2 -step" procedure is

$$
|\mathcal{R}|=\left|\mathcal{R}_{1}\right|+\left|\mathcal{R}_{2}\right| .
$$

Now, from proposition 3.3, we have $\left|\mathcal{R}_{1}\right| \leq \operatorname{rank}\left(\mathbf{H}_{\mathcal{E}}^{(1)}\right)$, with equality if and only if $\mathbf{H}_{\mathcal{E}}^{(1)}$ has $|\mathcal{E}|-\operatorname{rank}\left(\mathbf{H}_{\mathcal{E}}^{(1)}\right)$ columns equal to zero. Note that since $\operatorname{rank}\left(\mathbf{H}_{\mathcal{E}}\right)=n-k$, we must have $\operatorname{rank}\left(\mathbf{H}_{\mathcal{E}}^{(1)}\right)=\frac{n-k}{2}$. This defines the upper part of matrix $\mathbf{H}_{\pi(\mathcal{E})}$. Assume now that $\left|\mathcal{R}_{1}\right|=\operatorname{rank}\left(\mathbf{H}_{\mathcal{E}}^{(1)}\right)$ and let $\mathcal{E}^{\prime} \subset \mathcal{E}$ denotes the indices of the bits which have 
not been recovered after the first decoding step. We have $\left|\mathcal{E}^{\prime}\right|=\frac{n-k}{2}$. Since $\operatorname{rank}\left(\mathbf{H}_{\mathcal{E}}\right)=n-k$, we must have that $\operatorname{rank}\left(\mathbf{H}_{\mathcal{E}^{\prime}}\right)=\frac{n-k}{2}=\left|\mathcal{E}^{\prime}\right|$. Therefore, from proposition 3.3 all the remaining erased bits can be recovered by the second decoder.

On the other hand, if $\left|\mathcal{R}_{1}\right|<\operatorname{rank}\left(\mathbf{H}_{\mathcal{E}}^{(1)}\right)=\frac{n-k}{2}$, then $|\mathcal{R}|<n-k$ since $\left|\mathcal{R}_{2}\right| \leq \operatorname{rank}\left(\mathbf{H}_{\mathcal{E}}^{(2)}\right)=\frac{n-k}{2}$ from proposition 3.3 .

\section{Non-asymmetric DSC with turbo-codes}

In this section, we consider the problem of non-asymmetric SW coding based on turbo codes. In particular, we propose a coding procedure such that the $n-k$ last bits of $\mathbf{x}$ and $\mathbf{y}$ can be recovered by turbo decoding when the first $k$ bits are perfectly known.

We consider a code $\mathcal{C}$ which is made up of the parallel concatenation of two constituent codes $\mathcal{C}^{(1)}$ and $\mathcal{C}^{(2)}: \mathcal{C}^{(1)}$ is defined by a convolutional code with parity-check matrix $\mathbf{H}^{(1)}$ and $\mathcal{C}^{(2)}$ is defined by a parity-check matrix whose columns are a random permutation of the columns of $\mathbf{H}^{(1)}$. Due to the random nature of the code, optimal MAP block decoding is intractable and suboptimal turbo decoding has to be considered. We focus therefore on the same SW coding procedure as the one described in sections I-B and II but where the MAP decoding of both the error pattern $\mathbf{z}$ and the last $n-k$ bits of the sources (4) is approximated by a turbo decoding operation. We know from our derivations in section III-B that turbo decoding may fail recovering $n-k$ erasures if the parity-check matrix of the code does not have certain structural properties. In particular, considering the scenario where the first $k$ bits are perfectly known (i.e. $p=0$ ), we must have from proposition 3.4 that $\mathbf{H}_{\mathcal{E}}^{(1)}$ has $\frac{n-k}{2}$ columns equal to zero for successful decoding. In order to force this property, we propose the following syndrome computation procedure. Let $\mathbf{x}^{\prime} \triangleq\left[x_{1}, \ldots, x_{\frac{n+k}{2}}, 0, \ldots, 0\right]^{T}$ be a modified version of $\mathbf{x}$ where the last $\frac{n^{2}+k}{2}$ elements have been replaced by zero's. We define $\mathbf{s}_{x} \triangleq\left(\mathbf{s}_{x}^{(1)^{T}}, \mathbf{s}_{x}^{(2)^{T}}\right)^{T}$ where $\mathbf{s}_{x}^{(1)} \triangleq \mathbf{H}^{(1)} \mathbf{x}^{\prime}$, $\mathbf{s}_{x}^{(2)}=\mathbf{H}^{(2)} \mathbf{x}$. In fact, computing $\mathbf{s}_{x}^{(1)}$ from $\mathbf{x}^{\prime}$ rather than $\mathbf{x}$ is equivalent to forcing the last $\frac{n-k}{2}$ columns of $\mathbf{H}_{\mathcal{E}}^{(1)}$ to be equal to zero. Hence, the code satisfies the conditions of proposition 3.4 and the last $n-k$ bits of $\mathbf{x}$ can be recovered by turbo decoding when $p=0$.

The performance achieved by this approach is represented by the "dashed-dotted" curve in Fig. 5 for $n=2010$. The results are an average over different points of the SW region (i.e. different values of $k^{\prime}$ ). As for convolutional codes, we note that the soft decoding limits the error propagation phenomena and improve the performance with respect to the "matrixinversion" method.

\section{CONCLusions}

In this paper, we consider the problem of non-asymmetric distributed source coding. We propose a decoding algorithm which is robust to the errors which can occur in the estimation of the error pattern. In the case of SW coding based on turbo

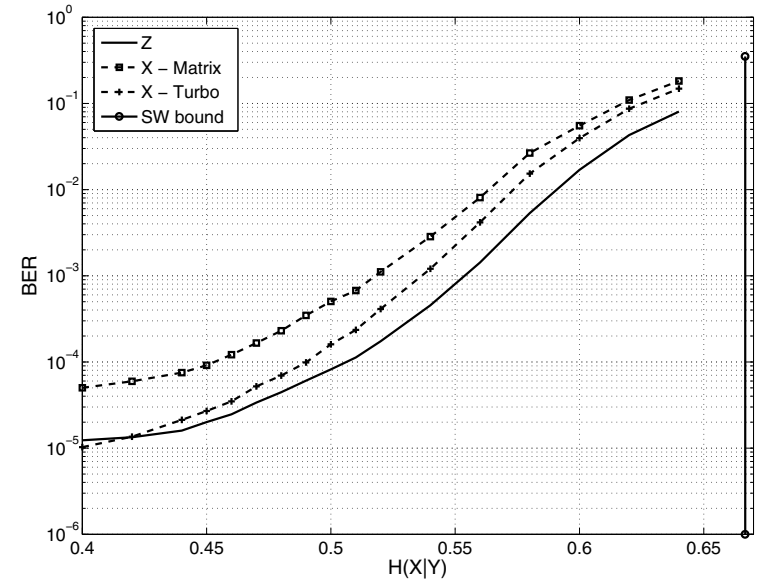

Fig. 5. Error propagation in the estimation of the source $X$. The BER achieved with different values of $k^{\prime}$ are averaged. The constituent convolutional codes are defined by parity-check polynomials $\left(\begin{array}{lll}15 / 17 & 1 & 0 \\ 15 / 17 & 0 & 1\end{array}\right)$.

codes, we derive code design rules so that turbo decoding can recover the last $n-k$ bits of the sources under limit conditions (i.e. $p=0$ ).

\section{REFERENCES}

[1] R. G. Gallager. Information Theory and Reliable Communication. Wiley, 1968.

[2] N. Gehrig and P.L. Dragotti. Symmetric and asymmetric slepianwolf codes with systematic and non-systematic linear codes. IEEE Communications Letters, 9(1):61-63, Jan. 2005.

[3] A.D. Liveris, Z. Xiong, and C.N. Georghiades. Compression of binary sources with side information at the decoder using LDPC codes. IEEE Communications Letters, 6(10):440-442, Oct. 2002.

[4] S. S. Pradhan and K. Ramchandran. Distributed source coding using syndromes (DISCUS): Design and construction. Proceedings of the IEEE International Data Compression Conference (DCC), 0(0):158-167, Mar. 1999.

[5] S. S. Pradhan and K. Ramchandran, "Distributed source coding: symmetric rates and applications to sensor networks," in Proceedings of the IEEE Data Compression Conference, March 2000, pp. 363-372.

[6] T. Richardson and R. Urbanke, Modern Coding Theory. Cambridge University Press, 2008.

[7] A. Roumy, K. Lajnef, and C. Guillemot. Rate-adaptive turbo-syndrome scheme for Slepian-Wolf coding. Proceedings of the IEEE Asilomar Conference on Signals, Systems, and Computers, vol. 0, Nov. 2007.

[8] A. Roumy and D Gesbert, "Optimal matching in wireless sensor networks," IEEE Journal on Selected Topics in Signal Processing, Special issue on Convex Optimization Methods in Signal Processing, December 2007.

[9] D. Schonberg, K. Ramchandran and S.S. Pradhan. Distributed code constructions for the entire Slepian-Wolf rate region for arbitrarily correlated sources Proceedings of the IEEE International Data Compression Conference (DCC), 0(0):292-301, Mar. 2004.

[10] D. Slepian and J.K. Wolf. Noiseless Coding of Correlated Information Sources. IEEE Transactions on Information Theory, vol. IT-19, pp. 471480, July 1973.

[11] V. Stankovic, A. D. Liveris, Z. Xiong, and C. N. Georghiades, "Design of slepian-wolf codes by channel code partitioning," in Proceedings of the IEEE Data Compression Conference, 2004, pp. 302-311.

[12] P. Tan and J. Li. A practical and optimal symmetric Slepian-Wolf compression strategy using syndrome formers and inverse syndrome formers. Proceeding of 43rd Annual Allerton Conference on Communication, Control and Computing, Sept. 2005.

[13] Z. Tu, J. Li, and R. S. Blum. An efficient SF-ISF approach for the Slepian-Wolf source coding problem. Eurasip Journal on Applied Signal Processing, 6(0):961-971, May 2005.

[14] A. Wyner. Recent results in the Shannon theory. IEEE Transactions on Information Theory, 20(0):2-10, 1974. 